Research Article

\title{
Propeller Force-Constant Modeling for Multirotor UAVs from Experimental Estimation of Inflow Velocity
}

\author{
Gaurang Gupta $(\mathbb{D})$ and Shaaban Abdallah \\ Department of Aerospace and Engineering Mechanics, University of Cincinnati, Cincinnati, OH 45221, USA \\ Correspondence should be addressed to Gaurang Gupta; guptagg@mail.uc.edu
}

Received 11 July 2017; Revised 6 November 2017; Accepted 6 December 2017; Published 10 April 2018

Academic Editor: Angel Velazquez

Copyright (c) 2018 Gaurang Gupta and Shaaban Abdallah. This is an open access article distributed under the Creative Commons Attribution License, which permits unrestricted use, distribution, and reproduction in any medium, provided the original work is properly cited.

\begin{abstract}
Design and simulation of an unmanned aerial vehicle (UAV) highly depends on the thrust produced by a motor-propeller combination. The aim of this paper is to model a generalized mathematical relationship between the motor RPM and the corresponding thrust generated for the preliminary design process of low Reynold's number applications. A method is developed to determine a generalized mathematical model which relates inflow velocity to coefficient of thrust using experimental data from 291 motor-propeller data points, comprising of input RPM and corresponding output thrust. Using this relationship, the Force Constant is calculated, which defines each Thrust-RPM mathematical model. In the first part, expression of the inflow ratio obtained from Blade Element and Momentum Theory (BEMT) is approximated to a simplified form. In the later part, the proposed mathematical model is validated against two new sets of pairs of motor-propeller combinations. A special note in the Appendix talks about the application of this mathematical model. The computed results are found to be in good agreement with the experimental data.
\end{abstract}

\section{Introduction}

The last few decades have shown an increase in the usage and development of UAVs. Most of today's UAVs used for reconnaissance, surveillance, and disaster management missions are powered using an electrical propulsion system which has shown significant improved propulsion efficiency and noise reduction over conventional combustion engine systems. UAVs have proven themselves very useful during the time of disaster management, where they hover in one place to securely drop medical supplies or get visuals of places $[1,2]$.

Energy density is defined as the amount of energy that a substance or component can store or transform per unit mass of itself. Fuel, which can be burnt, has an energy density of the order of magnitude three to four times more than that of solid-state fuel cell storage like a lithium polymer (LiPo) battery $[3,4]$. But the downside of using an engine is that it is highly inefficient and creates a lot of pollution. As studies suggest that the brushless direct-current (BLDC) motors are highly efficient, their usage has become very common in electric UAVs. As the propulsion system of an electric UAV consists of batteries, electronic speed controllers (ESC), motors, propellers, and so forth, a general survey shows that the weight of the propulsion system can account for approximately $50 \%$ of the total weight of the entire system as shown in [5-7]. Examples can also be taken from [8-10]. Thus, the optimization of the propulsion system of a UAV becomes a very crucial aspect of UAV design. There can be many other components in the propulsion system but the battery, motor, and propeller have a far more significant impact on the overall system. The other important aspect is to stabilize the UAV. Most of the general control methods are based on thrust force and the angular velocity of the motor assuming a simple parabolic relation. The performance and characteristics of the vehicle depend on the strong interaction between them. The purpose of this paper is not to present an optimization method or an optimal study but rather to study the interaction between these components and generalize them. The results of this paper have a significant application scope in the preliminary design process of any UAV (small or medium sized). This will help save time and drive the costs 
of the project down by eliminating the necessity of numerous amounts of initial bench tests using different propellers and motors to determine the best combination of propulsion system for the vehicle.

The method structured in this paper starts with estimating the inflow velocity which is simplified using approximations. There exists an alternative approach to estimate the inflow velocity. Commonly known as the 3/4 thumb rule, the geometric characteristics of the propeller are considered at 3/4 radius of the propeller from the center. The major parameters considered are the twist angle, the width of the propeller, and the airfoil section at that point. All these parameters will have to be calculated or measured from the actual propeller as these specifications are not available in off-the-shelf specifications. The advantage of the method discussed in this paper is that it uses only the parameters which exist in off-the-shelf available specifications like propeller diameter and pitch.

The first part of the paper discusses the development method and techniques utilized to simplify the conventional relation between the thrust generated by a propeller and its given RPM which is derived from very famous theories, namely the Blade Element and Momentum Theory. The next section discusses the assumptions undertaken to simply the estimation of inflow velocity which is the most important component in estimating the thrust generated by a propeller, using the existing relation. Secondly, the method used in the paper ensures that the output thrust is a function of only those parameters or a propeller that are available in any off-theshelf propeller and not the geometric parameters which are used to design a propeller. The later sections demonstrate the validation of the proposed model and the estimates of error induced due to simplification of the original coefficient of thrust versus RPM relation.

\section{Propeller Static Thrust-RPM Modeling}

Most of the propeller designs are based on the work of Betz mentioned in $[11,12]$. The design principle is based on optimizing the propeller's geometry for a certain specific operating condition such that the power required for that operation is minimized or can also be understood as maximizing the thrust generated for the given power. The thrust estimation model for a propeller in this study is based on the very famous Blade Element and Momentum Theory (BEMT). This theory helps in estimating the aerodynamic loads developed on the propeller, which can be used to estimate the thrust generated at a given RPM.

2.1. Thrust Model. First, we estimate $\mathrm{C}_{T}$ using the axial momentum theory $[13,14]$. The major assumptions are as follows: (i) no rotational motion is imparted to the flow by the propeller disk; (ii) the Mach number is small, so the fluid can be assumed to be incompressible; and (iii) the flow is steady as the propeller is assumed to be a thin disk (of cross-section area $A$ ), through which air passes, and the induced velocity is assumed to be constant at all points which lie on the same radius.

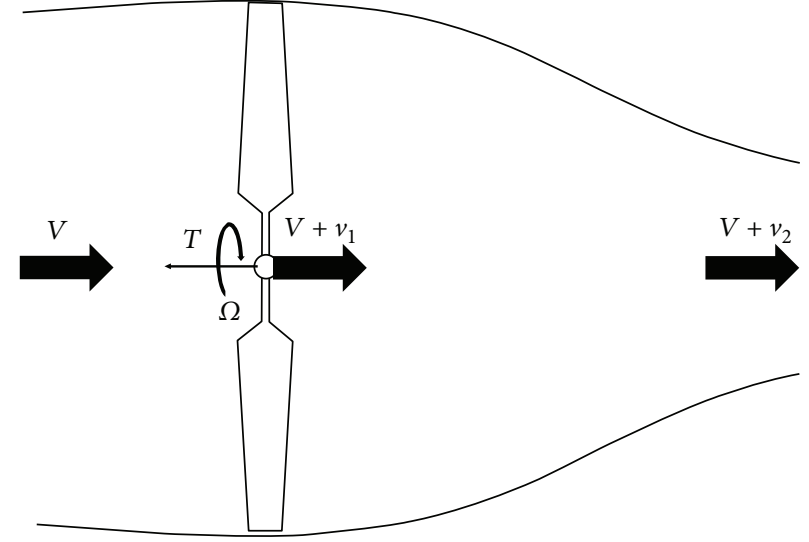

FIGURE 1: Propeller slipstream with axial velocity representation.

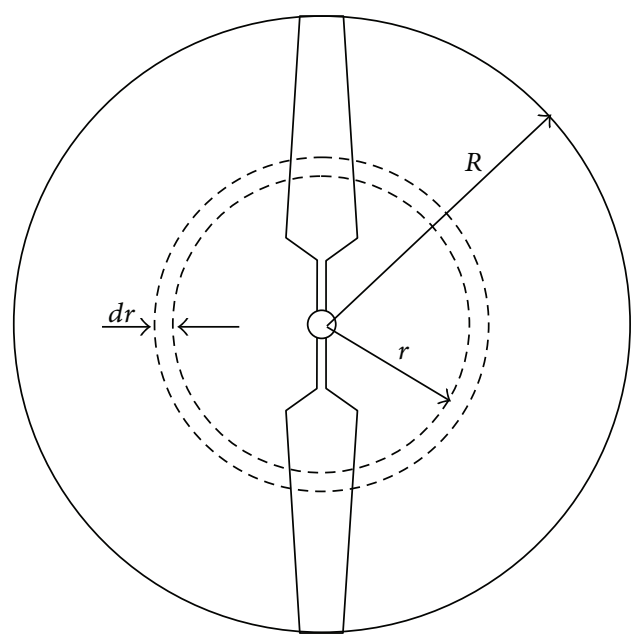

FIGURE 2: The propeller as a rotor disk.

A simplified model of a propeller stream tube is shown in Figure 1. The disk is assumed to be uniformly loaded, and the velocity of air across the rotor disk is $V+v_{1}$, which is assumed to be uniform across the disk and has the same magnitude before and after the disk [15]. On the rotor disk (Figure 2), at radius $r$ from the center, consider a ring with infinitesimal thickness $d r$. It is assumed that this elemental area of the disk is uniformly loaded. Thus, the elemental thrust coefficient, $d C_{T}$, that is, the nondimensional form of thrust for this elemental area, is

$$
d C_{T}=4\left(\lambda_{c}+\lambda_{i}\right) \lambda_{i} \bar{r} d \bar{r}
$$

where inflow ratio $\lambda_{i}$ is defined as the ratio of inflow velocity $v_{1}$ to tip velocity $\Omega R$; similarly, $\lambda_{c}$ is defined as the ratio of freestream velocity $V$ to tip velocity $\Omega R$ and $\bar{r}$ as $r / R$. Detailed derivation to obtain $d C_{T}$ is explained in Appendix A.

The modern propellers have a complex geometry, where the chord, pitch angle, and airfoil geometry vary along the radius of the blade to improve propeller efficiency. As the aerodynamic forces on a fixed wing (propeller blade) are 
governed by the rotating velocity, the effects of this can be described using a more fundamental Blade Element Theory [16-18], which takes into consideration the geometry of the propeller and the operating conditions. This theory assumes that each section of the wing acts as a $2 \mathrm{D}$ airfoil to produce aerodynamic forces (lift and drag as shown in Figure 3) which are then resolved to give elemental thrust per unit span.

Considering $N_{b}$ as the number of propeller blades, total elemental thrust coefficient at radius $r$ (Figure 2) can be defined as

$$
d C_{T}=\frac{N_{b} c}{R}\left[\theta \bar{r}^{2}-\left(\lambda_{c}+\lambda_{i}\right) \bar{r}\right] d \bar{r},
$$

where $\theta=\tan ^{-1}(p / \pi d)$. Detailed derivation to obtain $d C_{T}$, using Blade Element Theory, is explained in Appendix A.

On relating (1) and (2), derived elemental thrust coefficients from two different theories, we get

$$
\begin{aligned}
4\left(\lambda_{c}+\lambda_{i}\right) \lambda_{i} \bar{r} d \bar{r} & =\frac{N_{b} c}{R}\left[\theta \bar{r}^{2}-\left(\lambda_{c}+\lambda_{i}\right) \bar{r}\right] d \bar{r}, \\
\lambda_{i}^{2}+\left(\lambda_{c}+k\right) \lambda_{i}+\left(k \lambda_{c}-k \theta \bar{r}\right) & =0,
\end{aligned}
$$

where $k=N_{b} c / 4 R$ or $k=N_{b} c / 2 d$. Solving (4) for $\lambda_{i}$ and considering only a positive value, we get

$$
\lambda_{i}=-\frac{\lambda_{c}+k}{2}+\frac{1}{2} \sqrt{\left(\lambda_{c}-k\right)^{2}+4 k \theta \bar{r}}
$$

for hover, $\lambda_{c}=0$; (2) and (5) can be written as

$$
\begin{aligned}
d C_{T} & =4 k\left[\theta \bar{r}^{2}-\lambda_{i} \bar{r}\right] d \bar{r}, \\
\lambda_{i} & =-\frac{k}{2}+\frac{1}{2} \sqrt{k^{2}+4 k \theta \bar{r}} .
\end{aligned}
$$

2.2. Inflow Velocity Estimation. From geometric data collected using nine propellers (refer to Appendix B) $[19,20]$, at the root, $\theta$ ranges from $\theta_{\text {root,max }} \approx 0.48 \mathrm{rad}$, for high-pitchtype propellers, and $\theta_{\text {root,min }} \approx 0.11 \mathrm{rad}$, for low-pitch-type propellers, to $\theta_{\text {tip,avg }} \approx 0.07 \mathrm{rad}$ towards the tip. The variation of the twist angle with the radius can be a high-order polynomial but is assumed to be linear here. Figure 4 shows the variation of the pitch along the radius from root to tip, between high-pitch-type and low-pitch-type propellers. The geometric data collected from the nine propellers shows that the average effective radius of the blade that produces lift is approximately $86 \%$ of the total propeller radius, which includes hub losses.

If we consider infinite propellers in a given regime of diameter varying from 4 inches to 12 inches, the probability of occurrence of each point (where each point refers to a propeller with $\theta_{\text {root }}$ in between $\theta_{\text {root,max }}$ and $\theta_{\text {root,min }}$ ) in triangle $\mathrm{CDE}$ is equal. Thus, we try to estimate the average effect of $\theta_{\text {root }}$ over infinite possibilities such that in a broad domain, every propeller in this domain will have the same value of product of twist with varying radii shown as a red-shaded rectangle area in Figure 4. Here, it can safely be assumed that that infinite data set will have a normal distribution; that is, there will exist an equal number of propellers for

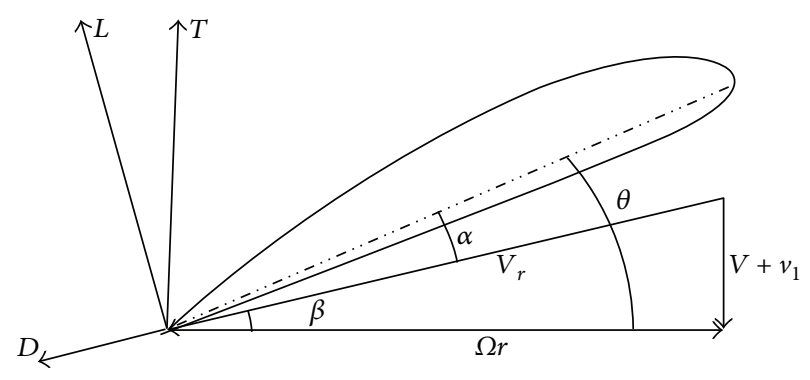

FIgURE 3: The blade cross-section showing simplified velocity triangle and aerodynamic forces.

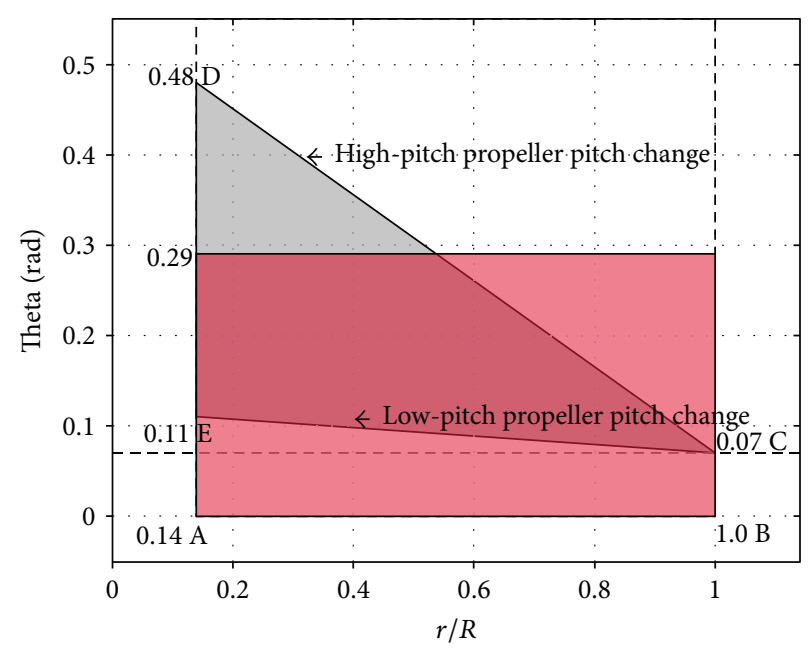

Figure 4: Twist variation along the blade radius.

all given values of $\theta_{\text {root }}$. Assuming a linear twist and normal distribution (considering equal probability) of data sets between $\theta_{\text {root,max }}$ and $\theta_{\text {root,min }}$ to $\theta_{\text {tip,avg, we calculate }}$ $(\theta \bar{r})_{\text {mean }}$ as below and represent it as a uniform pitch propeller (as shown by the red-shaded rectangular area)

$$
(\theta \bar{r})_{\text {mean }}=\frac{\operatorname{Area}(\mathrm{ABCE})+\operatorname{Area}(\mathrm{ABCD})}{2} \times\left.[\bar{r}]\right|_{0.14} ^{1} .
$$

Solving for $(8)$, we get $(\theta \bar{r})_{\text {mean }} \approx 0.25$, giving $4(\theta \bar{r})_{\text {mean }}=$ 1 and $\theta_{\text {mean }} \approx 0.29 \mathrm{rad}$. Using the above approximation, we assume that the inflow velocity across the entire rotor disk is uniform. From (7) $\lambda_{i}$ can thus be defined as

$$
\lambda_{i}=-\frac{k}{2}+\frac{1}{2} \sqrt{k^{2}+4 k(\theta \bar{r})_{\text {mean }}}
$$

From (8) and (9), we have a relation defined for $\lambda_{i}$ for hover defined as

$$
\lambda_{i}=-\frac{\sqrt{k^{2}+k}-\sqrt{k}}{2} .
$$

Considering that about $80 \%$ to $92 \%$ of the propeller blade diameter effectively produces lift, the analytical 
solution, considering diameter effectiveness $e_{d}$, for (6) is as follows:

$$
C_{T}=\int_{1-e_{d}}^{1} 4 k\left[\theta \bar{r}^{2}-\lambda_{i} \bar{r}\right] d \bar{r}
$$

From (10) and (11), we have

$C_{T}=\frac{4}{3} k \theta\left[1-\left(1-e_{d}\right)^{3}\right]-k(\sqrt{k(1+k)}-\sqrt{k})\left[1-\left(1-e_{d}\right)^{2}\right]$,

where $\theta=\tan ^{-1}(p / \pi d)$ and $k$ and $e_{d}$ are selected from Tables 1 and 2. Thrust is defined as

$$
T=C_{T} \rho \pi R^{2}(\Omega R)^{2} .
$$

The final Thrust-RPM mathematical model and Force Constant can be defined as

$$
T=k_{f} \Omega^{2}, \quad k_{f}=\frac{1}{6} \rho \pi R^{4} e_{d}^{4} C_{T},
$$

where $C_{T}$ can be calculated from (12) and $e_{d}$ is selected from Table 1.

It is well known that the entire propeller is not capable of producing lift as there always exists hub and tip losses. Thus, a range of values of effective diameters has to be approximated using the available geometric data of nine propellers (refer to Appendix B).

\section{Verification of the Mathematical Model}

Verification of the above mathematical model is done using 291 available experimental data points obtained from http://www.hobbyking.com, which consists of various motor propeller combinations. Each data point is described as the thrust generated at a given RPM for a set of a motor and propeller. 36 propellers are used ranging from 4-inch diameter to 16-inch diameter, and 18 different motors are used ranging from $360 \mathrm{kV}$ to $3100 \mathrm{kV}$. The air density used for the estimation of all data is $1.18 \mathrm{~kg} / \mathrm{m}^{3}$, and the typical $e_{d}$ values (from Table 1) were used to calculate (estimate) the thrust. A small MATLAB (R2016a version) script is used to calculate the thrust and generate all graphs.

The graphs in Figure 5 show the thrust estimated at different given RPM for few combinations (out of the 291 data points) of the motor and propeller in comparison to experimental data. These graphs are used to build and verify the proposed mathematical model. On all graphs, "o" points are the experimental thrust values at a given RPM and " $\Delta$ " points are the estimated thrust values from the proposed model for the respective given RPM. This gives us a better understanding of the comparison between the experimentally obtained thrust and the estimated thrust. It can be observed from the results that for typical $e_{d}$ values (from Table 1) used, the estimated thrust lies within the range of $\pm 10 \%$ of the experimental data. The four chosen propellers have pitch-to-diameter ratios varying from $1.125(4 \times 4.5$ prop) to $0.3375(16 \times 5.4$ prop). This ensures that the
TABLe 1: Estimated effective propeller diameter.

\begin{tabular}{lcc}
\hline$p / d$ & $e_{d}$ range & Typical $e_{d}$ \\
\hline$p / d<0.4$ & $0.90-0.92$ & 0.91 \\
$0.4 \leq p / d<0.8$ & $0.87-0.89$ & 0.88 \\
$0.8 \leq p / d<0.9$ & $0.82-0.86$ & 0.86 \\
$p / d \geq 0.9$ & $0.80-0.81$ & 0.80 \\
\hline
\end{tabular}

TABLE 2: Estimated $c / d$ ratio for $k$ calculation.

\begin{tabular}{lc}
\hline$d$ inch & $c / d$ \\
\hline 4 & 0.09 \\
$5-6$ & 0.1 \\
$7-9$ & 0.11 \\
$10-12$ & 0.12 \\
$13-14$ & 0.13 \\
$15-16$ & 0.14 \\
\hline
\end{tabular}

proposed mathematical model is valid for a wide range of propellers available and the validity of the proposed $e_{d}$ as a function of $p / d$.

It is clear from [21-24] that there are far too many assumptions made in deriving the Blade Element and Momentum Theory for axial flow across a propeller. To generalize the thrust-RPM relation, there were more assumptions made with respect to the geometry of the propeller and the flow across it. Thus, it becomes necessary to quantify the magnitude of error induced in the results in comparison to the experimental data. The results shown in Figure 6 are the individual estimated thrust values at a given RPM for a set of motor and propeller combinations. The graph consists of all the 291 studied data points, which shows a comparison between the collected experimental thrust data from different motor-propeller combinations with the estimated thrust data which is calculated using the proposed mathematical model. The graph shows a normal distribution of the error in estimation of the thrust. The error measured is given as

$$
\text { Error }=\left(\frac{T_{\text {est }}-T_{\text {exp }}}{T_{\exp }}\right) * 100 \text {, }
$$

where $T_{\text {est }}$ is the value of thrust estimated using the proposed mathematical model against $T_{\text {exp }}$, the experimental value of the thrust.

It can be calculated from the graph that more than $70 \%$ of the estimated values (shaded area in the graph) lie within a range of $\pm 10 \%$ of the experimental data. Few estimated values, which are off by more than $\pm 10 \%$, can be due to the lack of knowledge of the experimental setup data, experimental conditions (example air density), exact propeller geometry, airfoil characteristics, and so forth. 


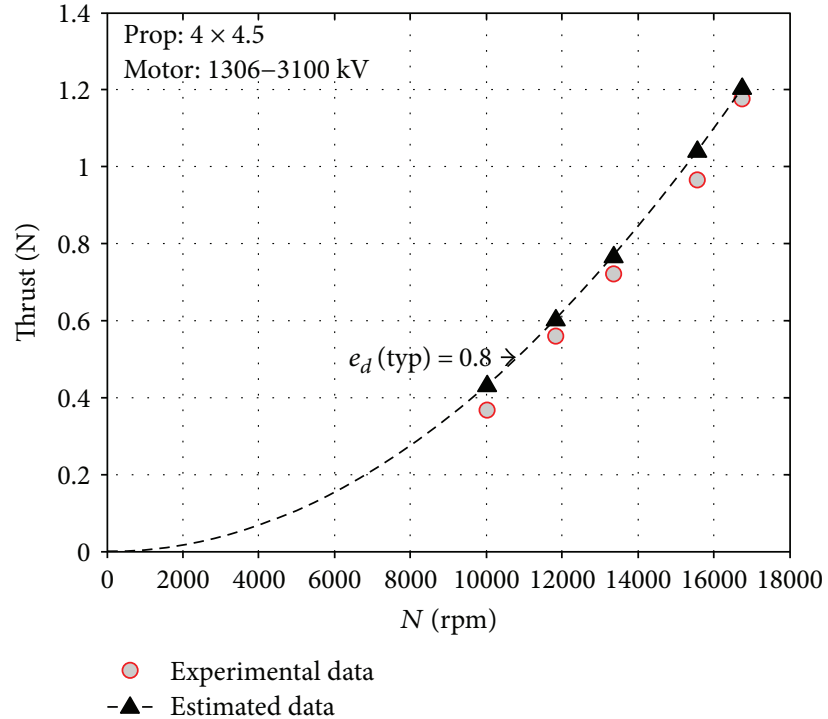

(a)

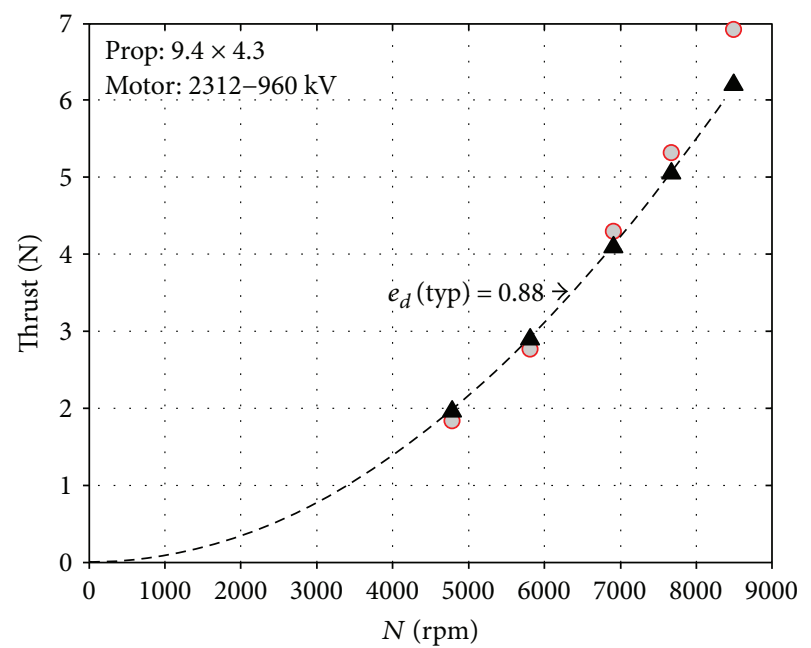

O Experimental data

- $\Delta$ - Estimated data

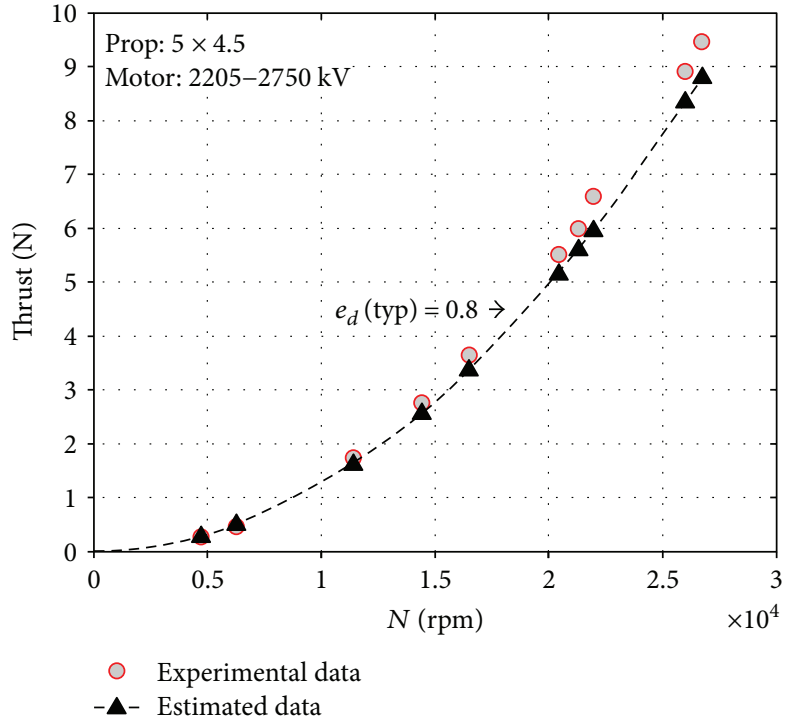

(b)

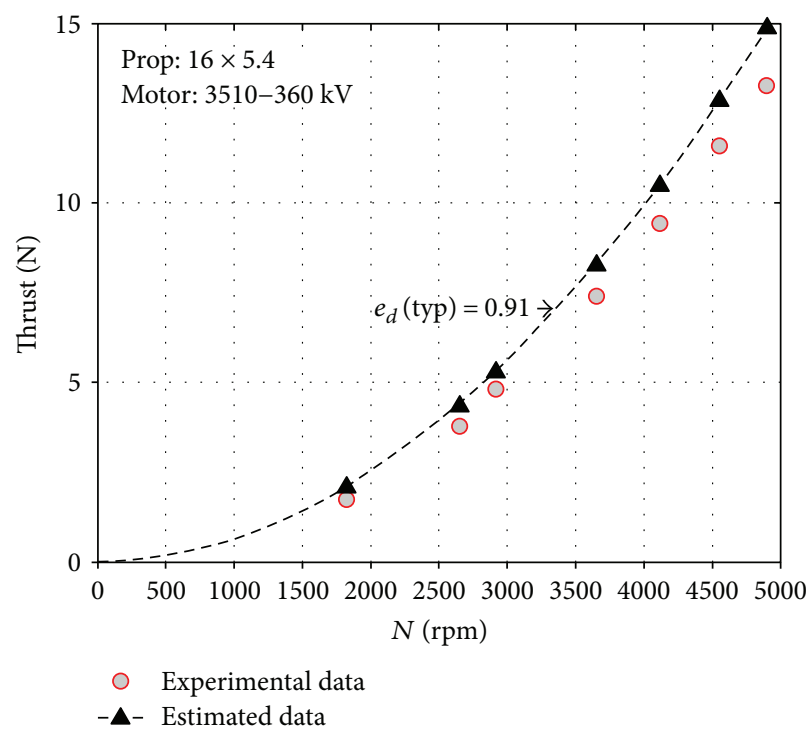

(d)

- - Estimated data

(c)

Figure 5: Verification for the proposed model using experimental data.

\section{Validation of the Mathematical Model}

As the verification of the proposed Thrust-RPM mathematical model was done using open source data available on the website https://hobbyking.com, it will be good practice to validate the proposed mathematical model using data published by different authors who have conducted such experiments. The proposed model is validated using two data sets published by two authors. In the first set (Figure 7(a)) taken from [25], we validate a model which uses a $10 \times 4.7$ propeller. The blade effectiveness factor, $e_{d}$, used is 0.89 , and the air density considered is $1.225 \mathrm{~kg} / \mathrm{m}^{3}$. It is observed that the estimated data at higher RPM is undercalculated. This implies that the assumed hub losses for the propeller are greater than what actually occurs in reality and the propeller is more efficient than estimated by the model.

In the second set (Figure 7(b)) taken from [10], we validate a model which uses a $16 \times 4$ propeller. The blade effectiveness factor of 0.92 is used, and the density of air considered is $1.225 \mathrm{~kg} / \mathrm{m}^{3}$. It is observed that the estimated data is undercalculated but pretty much matches with the experimental data as the estimated data lies within the $-10 \%$ limit of the experimental data. From these results, we can say that the proposed mathematical model of the propeller can estimate promising data.

The above estimations are also affected by the increase in Reynolds number at a higher RPM which alters the 


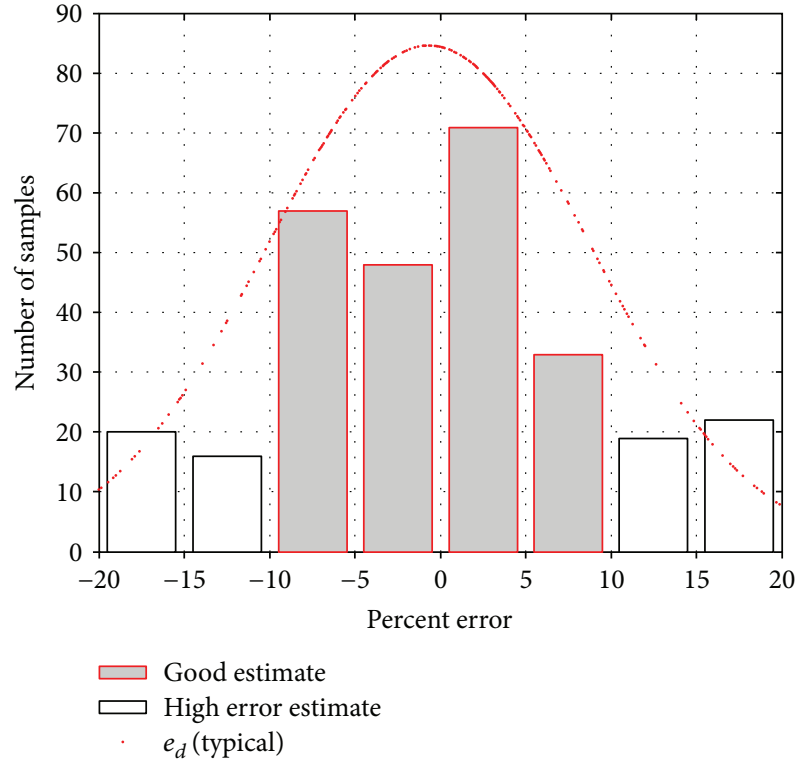

Figure 6: Comparison between the experimental thrust and estimated thrust for 291 samples.

airfoil characteristics $\left(C_{l}\right.$ and $\left.C_{d}\right)$; that is, the assumption of having a constant $C_{l}$ for the entire blade over the entire range of RPM is not always good for high RPM. But we will see in the later part of the paper that this undercalculation can be compensated for by adjusting for $e_{d}$.

\section{Conclusion}

As observed from all the above results, even though there will always be some discrepancy between the experimental data and the estimated data, the proposed mathematical model

$$
T=\frac{1}{16} \rho \pi R^{4} e_{d}^{4} C_{T} \Omega^{2}
$$

with $C_{T}$ defined as

$$
\begin{aligned}
C_{T}= & \frac{4}{3} k \theta\left[1-\left(1-e_{d}\right)^{3}\right] \\
& -k(\sqrt{k(1+k)}-\sqrt{k})\left[1-\left(1-e_{d}\right)^{2}\right],
\end{aligned}
$$

does give promising results. The simplification of the inflow velocity estimation is likely to introduce $\pm 10 \%$ error in the magnitude of thrust generated in the majority of cases. It is interesting to note how the effects of pitch variation and rotor diameter effectiveness is captured in the mathematical expression developed for $C_{T}$. Thus, this process is different from curve fitting as we cannot predict the change in thrust that can occur due to change in any other parameter in (16) by curve-fitted data. Figure 6 shows the discrepancies between the estimated data and the experimental data. It is important to note that this discrepancy occurs at high RPM where it becomes difficult to generalize the aerodynamic phenomenon over such a huge range of propeller sizes and RPM in this inefficient regime of operation. It was only possible to validate the above model using propellers ranging from 4-inch diameter to 16-inch diameter, the size range which is generally preferred for small-size UAVs. Appendix C discusses an application note of this mathematical model in a realworld scenario.

\section{Appendix}

\section{A. Blade Element and Momentum Theory}

General momentum theory is a magnificently huge theory which deals with the flow characteristics across the propeller disk at a much broader level than Blade Element Theory as discussed in [26] with all its development and assumptions around it. We first estimate $\mathrm{C}_{T}$ using momentum theory $[13,14]$. A simplified model of a propeller stream tube is shown in Figure 1. A uniformly loaded disk is considered and as it has been proved in $[27,28]$ that even for a uniformly loaded disk the axial velocity at the propeller disk is not the same throughout, we assume it to be the same for simplicity of the derivation, and we also consider that the axial velocity of the air across the rotor disk, $V+v_{1}$, has the same magnitude before and after the disk as considered in [15]. There are many more assumptions made in this theory which are talked about in detail in [21-23]. As mass is conserved, the mass flow rate across the disk is given as

$$
\dot{m}=\rho A\left(V+v_{1}\right)
$$

From Newton's Second Law, the rate of change of momentum can be defined as the resultant force applied on the airflow, that is, thrust, which can be defined as the product of the mass flow rate and the change in velocity

$$
T=\rho A\left(V+v_{1}\right)\left(V+v_{2}-V\right)=\rho A\left(V+v_{1}\right) v_{2}
$$

Assuming an ideal system, work done by thrust force per unit time equals the rate of change of translational kinetic energy of the airflow which can be defined as

$$
T\left(V+v_{1}\right)=\frac{1}{2} \dot{m}\left[\left(V+v_{2}\right)^{2}-V^{2}\right]
$$

Solving (A.3) for $v_{2}$, using the above derived relations, we get a relation between the far-field velocity in the slipstream and the inflow velocity at the disk as $v_{2}=2 v_{1}$. This relation is an alternative form of the well-known Froude's law which states that the axial velocity at the disk is the arithmetic mean between the one at the upstream and downstream (in the wake) infinity [29].

Considering a rotor disk, (Figure 2), at radius $r$ from the center, a ring with infinitesimal thickness $d r$, and considering the assumptions mentioned above about uniform disk 


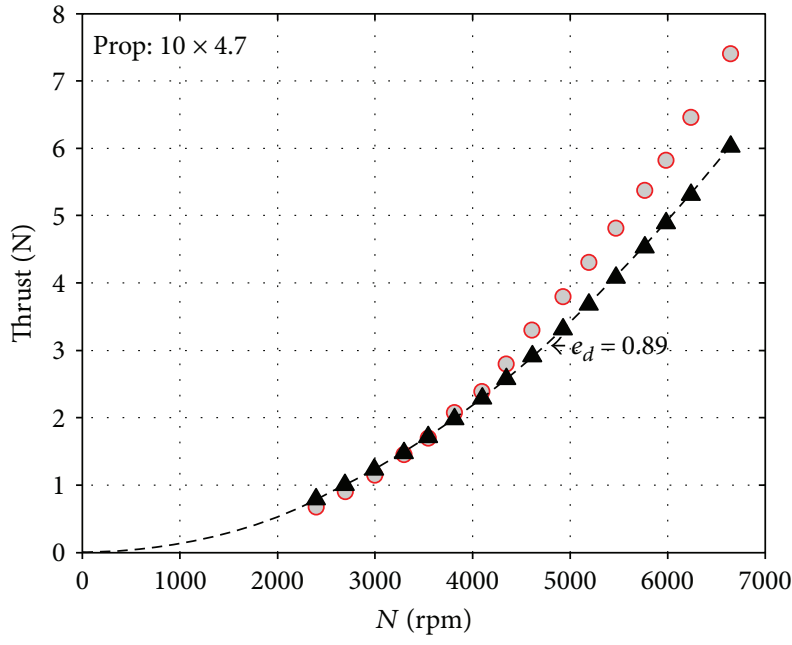

- Experimental data

- $\mathbf{\Delta -}$ Estimated data

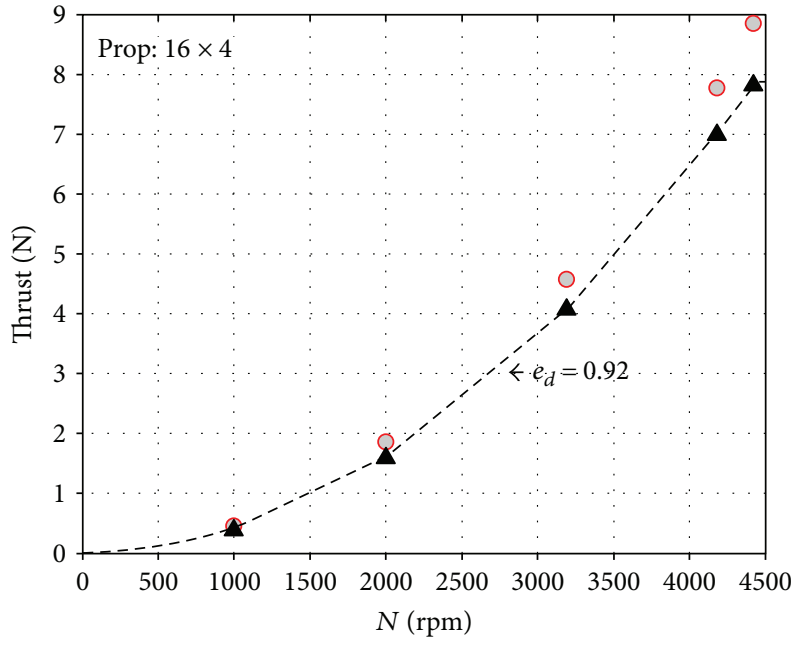

- Experimental data

- $\boldsymbol{\Delta}$ - Estimated data

(a)

(b)

FIGURE 7: Validation of the Thrust-RPM mathematical model.
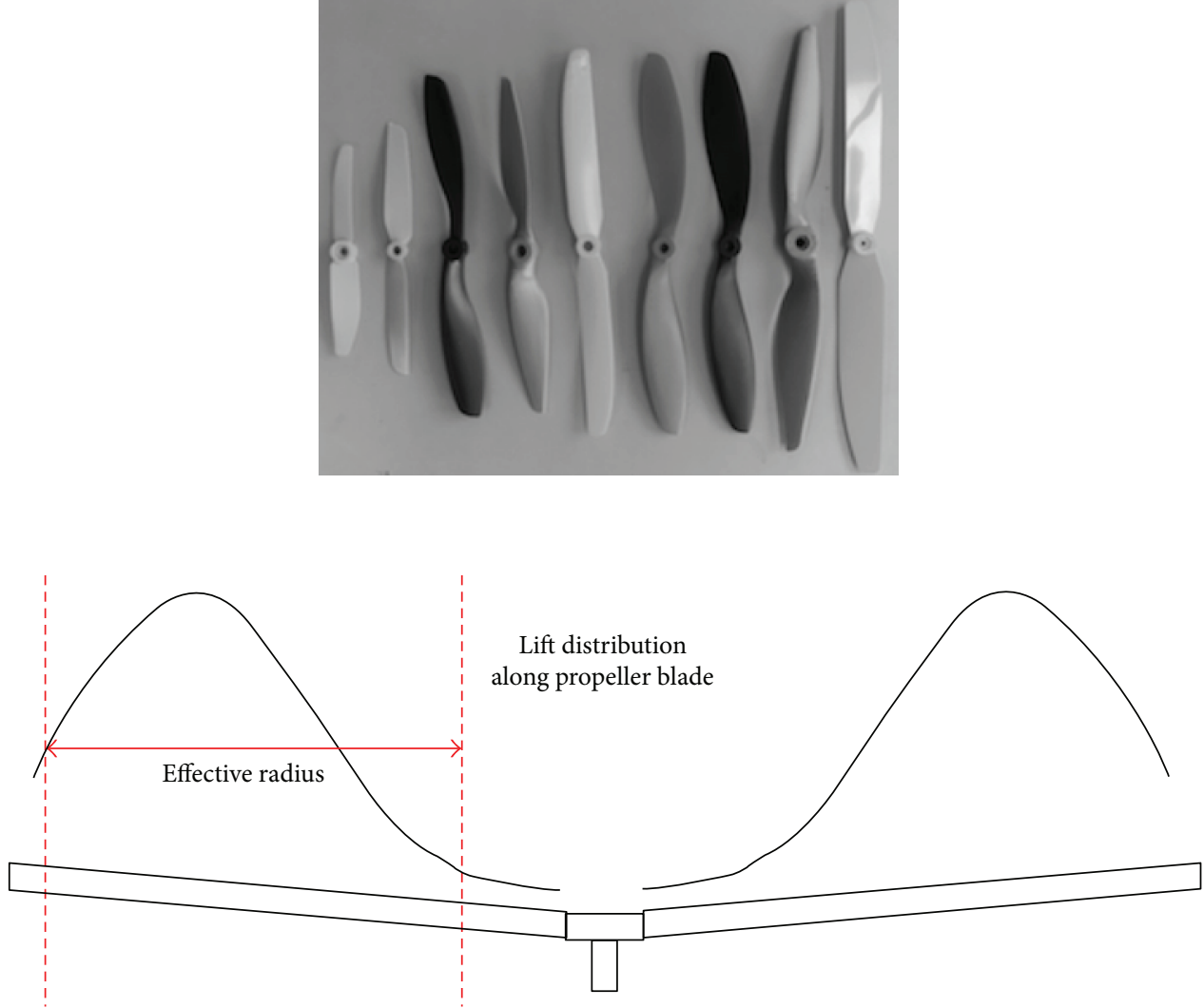

FIGURE 8: Reference propellers.

loading, the elemental thrust, $d T$, generated by this elemental area is described as

$$
d T=2 \rho\left(V+v_{1}\right) v_{1} d A=2 \rho\left(V+v_{1}\right) v_{1} 2 \pi r d r .
$$

Thus, the nondimensional form of the elemental thrust can be defined as

$$
d C_{T}=\frac{d T}{\rho \pi R^{2}(\Omega R)^{2}} .
$$




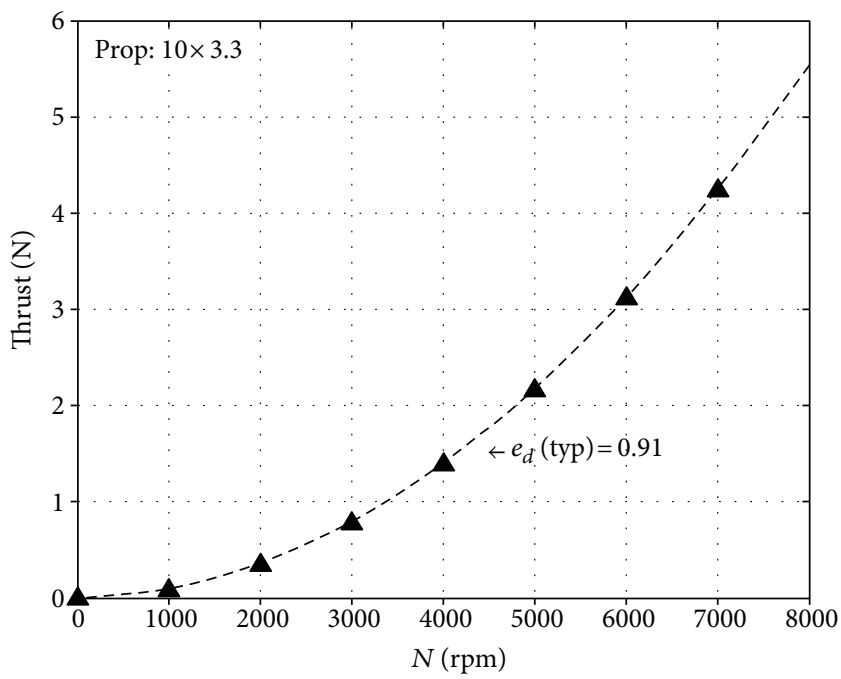

(a)

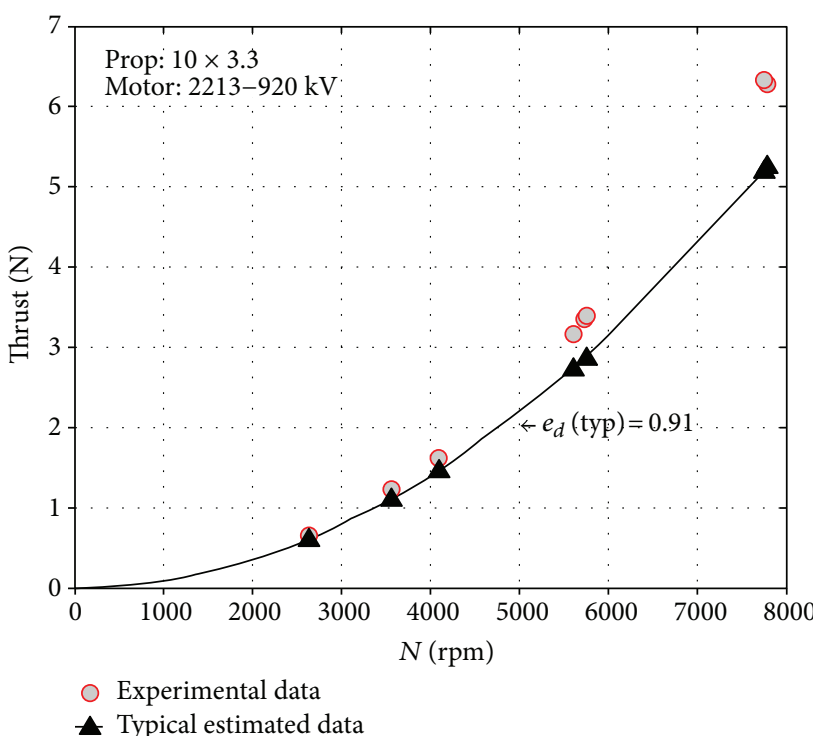

(b)

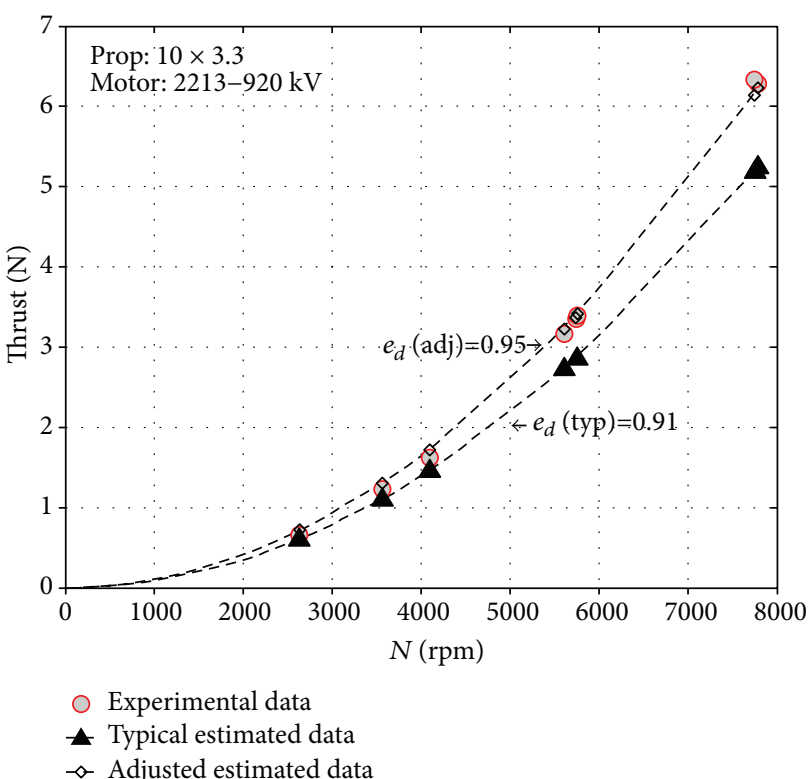

(c)

FIgURE 9: Fine tuning of the model based on the experimental data.

Solving (A.5) for $d T$, we get (1).

Using Blade Element Theory [11, 12], elemental aerodynamic forces, lift and drag per unit span are respectively given as

$$
\begin{aligned}
& d L=\frac{1}{2} \rho V_{r}^{2} c C_{l} d r \\
& d D=\frac{1}{2} \rho V_{r}^{2} c C_{d} d r
\end{aligned}
$$

which are resolved (Figure 3 ) to give the elemental thrust as

$$
d T=\frac{1}{2} \rho V_{r}^{2} c\left[C_{l} \cos \beta-C_{d} \sin \beta\right] d r
$$

where $V_{r}$ and $c$ are the relative velocity of air experienced by the blade and the chord of the blade at radius $r$, respectively [19] (Figure 3). $C_{l}$ and $C_{d}$ are lift and drag coefficients, respectively, for the propeller airfoil, considered to be the same, radially, for each cross-section throughout the blade. $\beta$ is the relative inflow angle defined as $\beta=\tan ^{-1}$ $\left(\left(V+v_{1}\right) / \Omega R\right)$. Assuming angle $\beta$ has very small magnitude, $\cos \beta \approx 1, \sin \beta \approx \beta$, and $\beta \approx\left(V+v_{1}\right) / \Omega R$. Substituting these values in (A.8) we get

$$
d T=\frac{1}{2} \rho V_{r}^{2} c\left[C_{l}-C_{d} \beta\right] d r .
$$

Further, considering thin airfoil theory [14] and airfoil data to be origin centered, the product, $C_{d} \beta$, is assumed to be very small and the lift curve slope can be approximated 
as $\mathrm{C}_{l \alpha} \approx 2 \pi$, where $\alpha=(\theta-\beta)$ and $\theta$ is the blade twist. With these assumptions, (A.9) can be simplified to

$$
d T=\frac{1}{2} \rho V_{r}^{2} c\left[C_{l_{\alpha} \alpha}\right] d r=\frac{1}{2} \rho V_{r}^{2} c[2 \pi(\theta-\beta)] d r .
$$

Next, considering $\left(V+v_{1}\right)^{2} \ll(\Omega R)^{2}$ gives $V_{r}^{2} \approx(\Omega r)^{2}$. Using these approximations, we can now define the elemental thrust as

$$
d T=\rho \pi(\Omega r)^{2} c\left(\theta-\frac{V+v_{1}}{\Omega r}\right) d r
$$

From (A.5) and (A.11), the elemental thrust coefficient at radius $r$ can be given as (2).

\section{B. Propeller Geometrical Data Approximation}

Nine propellers are shown in Figure 8, which range from 4inch to 12-inch diameters, and are used to get geometric data like twist angle, $c / d$ ratio, and effective diameter of the propellers. An approximate effective diameter is measured by eliminating the propeller hub and thin tip section. The relation $\theta=\tan ^{-1}(p / \pi d)$ suggests that $\theta_{\max }$ will occur for the maximum value of the $p / d$ ratio and vice versa is true for $\theta_{\text {min }} \cdot \theta_{\text {root,max }}$ and $\theta_{\text {root,min }}$ are thus calculated using a $4 \times 4.5$ (leftmost) propeller and $12 \times 4$ (rightmost) propeller, respectively. The values calculated for $\theta_{\text {root,max }}$ and $\theta_{\text {root,min }}$ are 0.34 rad and $0.11 \mathrm{rad}$, respectively.

If a propeller is carefully observed, the blade section near the hub and the tip is tapered to the extent where it becomes incapable of generating any significant amount of lift. Based on these observations and measurements made (of diameter with significant lift-generating area), a few typical effective diameter, $e_{d}$, values are proposed. There may exist extreme cases where the value of $e_{d}$ falls out of the proposed range, and one such case is discussed in Appendix C.

\section{Application Note}

Let us consider a design case where we have concluded that the propeller suitable for a UAV is a $10 \times 3.3$-inch propeller to be used with some low-kV motor (typically less than $1000 \mathrm{kV}$ ). Using the proposed Thrust-RPM mathematical model, we have the following thrust versus RPM characteristic curve as shown in Figure 9(a), plotted using a typical value of $e_{d}$. From the validation, we can say there is a high probability of the estimated data to be within $\pm 10 \%$ of the actual values. But before the final design, the experiments conducted give the following data set at discrete points (Figure 9(b)) which can be used to fine-tune the parameters in the proposed model as shown in Figure 9(c).

If we look at the mathematical model carefully, we can understand the parameters which vary from system to system. The value of the thrust is directly influenced by $C_{T}$ and $\rho . C_{T}$ is a function of $k, \theta$, and $e_{d}$. Further, $k$ is a function of the ratio $c / d$. The parameters which have been highly approximated in the proposed model are $k$ and $e_{d}$. Is it suggested that the tuning of the model should be done in the following way:

(1) Start with using the corrected value of air density.

(2) Next, try to change $e_{d}$; the effective radius of a good propeller will lie between 0.75 and 0.95 .

(3) Lastly, if the expected value of $e_{d}$ lies outside the above-mentioned range, try to adjust $k$.

By following the above procedure, we end up with a finely tuned thrust-RPM model of the propeller as shown in Figure 9(c).

\section{Nomenclature}

$\begin{array}{ll}A: & \text { Rotor disk area } \\ \alpha: & \text { Angle of attack } \\ \beta: & \text { Relative inflow angle } \\ c: & \text { Chord } \\ C_{T}: & \text { Thrust coefficient } \\ d: & \text { Propeller diameter } \\ d D: & \text { Elemental drag per unit span } \\ e_{d}: & \text { Diameter effectiveness } \\ \lambda_{i}: & \text { Inflow ratio } \\ \lambda_{c}: & \text { Flow ratio } \\ d L: & \text { Elemental lift per unit span } \\ k_{f}: & \text { Motor-propeller Force Constant } \\ \dot{m}: & \text { Mass flow rate of air } \\ N: & \text { Rotation per minute } \\ N_{b}: & \text { Number of propeller blades } \\ \Omega: & \text { Propeller angular velocity rad/s } \\ p: & \text { Propeller pitch } \\ R: & \text { Rotor radius from hub to tip } \\ d r: & \text { Incremental radius } \\ \rho: & \text { Air density } \\ T: & \text { Propeller thrust } \\ \theta: & \text { Blade twist angle } \\ V: & \text { Free-stream velocity } \\ V_{r}: & \text { Relative velocity } \\ v_{1}: & \text { Inflow velocity } \\ v_{2}: & \text { Increment in far-field velocity. }\end{array}$

\section{Conflicts of Interest}

The authors declare that there is no conflict of interest regarding the publication of this paper.

\section{Acknowledgments}

The author would like to thank Shivani Chandak, Gargi Kadoo, and Kiran Siddappaji (University of Cincinnati) for their comments that greatly improved the manuscript. The authors would also like to show their gratitude to Ted Baldwin and Bryan Brown from the University of Cincinnati for helping them with reference papers and for providing propellers for this research, respectively. 


\section{References}

[1] S. R. Kesler, Propeller thrust analysis using Prandtl's lifting line theory, a comparison between the experimental thrust and the thrust predicted by Prandtl's lifting line theory [M. S. thesis], Dept. of Mechanical Engineering, The University of Utah, Salt Lake City, UT, USA, 2014.

[2] C. Tjhai, Developing stochastic model of thrust and flight dynamics for small UAVs [M. S. Thesis], The University of Minnesota, Minneapolis, MN, USA, 2013.

[3] P. Lindahl, E. Moog, and S. R. Shaw, "Simulation, design, and validation of an UAV SOFC propulsion system," IEEE Transactions on Aerospace and Electronic Systems, vol. 48, no. 3, pp. 2582-2593, 2012.

[4] O. Atlam and M. Kolhe, "Performance evaluation of directly photovoltaic powered DC PM (direct current permanent magnet) motor-propeller thrust system," Energy, vol. 57, pp. 692698, 2013.

[5] M. J. Stepaniak, F. van Grass, and M. U. De Haag, "Design of an electric propulsion system for a quadrotor unmanned aerial vehicle," Journal of Aircraf, vol. 46, no. 3, pp. 1050-1058, 2009.

[6] P. Ragot, M. Markovic, and Y. Perriard, "Optimization of electric motor for a solar airplane application," IEEE Transactions on Industry Applications, vol. 42, no. 4, pp. 1053-1061, 2006.

[7] O. Gur and A. Rosen, "Optimizing electric propulsion systems for unmanned aerial vehicles," Journal of Aircraft, vol. 46, no. 4, pp. 1340-1353, 2009.

[8] D. A. Lawrence and K. Mohseni, "Efficiency analysis for longduration electric MAVs," in Infotech@Aerospace Conference, pp. 1649-1661, Arlington, Virginia, USA, September 2005.

[9] T. McElory and D. Brian Landrum, "Simulated high-altitude testing of a COTS electric UAV motor," in 50th AIAA Aerospace Sciences Meeting including the New Horizons Forum and Aerospace Exposition, Nashville, TN, USA, January 2012.

[10] D. Kaya, A. Tevfik, A. T. Kutay, and O. Tekinalp, "Design and control of a micro UAV," in AIAA Atmospheric Flight Mechanics Conference, AIAA AVIATION Forum, (AIAA 2016-3857), Washington, D.C., USA, June 2016.

[11] H. Amini and S. Steen, "Experimental and theoretical analysis of propeller shaft loads in oblique inflow," Journal of Ship Research, vol. 55, no. 4, pp. 268-288, 2011.

[12] J. H. Horlock, Actuator Disk Theory, McGraw-Hill, New York, NY, USA, 1978, Chaps 2, 3.

[13] W. F. Phillips, "Propeller momentum theory with slipstream rotation," Journal of Aircraft, vol. 39, no. 1, pp. 184-187, 2002.

[14] M. Amini, L. Sileo, and S. Steen, "Numerical calculations of propeller shaft loads on azimuth propulsors in oblique inflow," Journal of Marine Science and Technology, vol. 17, no. 4, pp. 403-421, 2012.

[15] K. Siddappaji and M. Turner, "Counter rotating propeller design using blade element momentum theory," in Proceedings of the 22nd ISABE Conference, Phoenix, AZ, USA, 2015.

[16] R. Martinez-Alvarado, F. J. Ruiz-Sanchez, A. Sanchez-Orta, and O. Garcia-Salazar, "Dynamic response of BLDC-thruster for small scale quadrotors under aerodynamic load torque," in 2014 IEEE International Autumn Meeting on Power, Electronics and Computing (ROPEC), pp. 163-176, Ixtapa, Mexico, 2014.

[17] H. Matthew and J. W. Gregory, "Blade element momentum modeling of low-Re small UAS electric propulsion systems," in 33rd AIAA Applied Aerodynamics Conference, AIAA AVIATION Forum, (AIAA 2015-3296), Dallas, TX, USA, June 2015.
[18] B. W. McCormick, "Blade element theories," in Aerodynamics of VISTOL Flight, , pp. 79-93, Dover, NY, USA, 1999.

[19] R. W. Deters and M. S. Selig, "Static testing of micro propellers," in 26th AIAA Applied Aerodynamics Conference, Guidance, Navigation, and Control and Co-located Conferences, Honolulu, HI, USA, August 2008.

[20] H. Otsuka and K. Nagatani, "Effect of Reynolds numbers of 10,000 to 100,000 on rotor blades of small unmanned aerial vehicles," in 34th AIAA Applied Aerodynamics Conference, AIAA AVIATION Forum, (AIAA 2016-3423), Washington, D.C., USA, June 2016.

[21] R. Bontempo and M. Manna, "Analysis and evaluation of the momentum theory errors as applied to propellers," AIAA Journal, vol. 54, no. 12, pp. 3840-3848, 2016.

[22] R. Bontempo and M. Manna, "Highly accurate error estimate of the momentum theory as applied to wind turbines," Wind Energy, vol. 20, no. 8, pp. 1405-1419, 2017.

[23] R. Bontempo and M. Manna, "Effects of the approximations embodied in the momentum theory as applied to the NREL PHASE VI wind turbine," International Journal of Turbomachinery Propulsion and Power, vol. 2, no. 2, p. 9, 2017.

[24] H. A. Madsen, R. Mikkelsen, S. Øye, C. Bak, and J. Johansen, "A detailed investigation of the Blade Element Momentum (BEM) model based on analytical and numerical results and proposal for modifications of the BEM model," Journal of Physics: Conference Series, vol. 75, 2007.

[25] W. Khan and M. Nahon, "A propeller model for general forward flight conditions," International Journal of Intelligent Unmanned Systems, vol. 3, no. 2/3, pp. 72-92, 2015.

[26] J. N. Sørensen, General Momentum Theory for Horizontal Axis Wind Turbines, Springer, Switzerland, 2016.

[27] J. N. Sørensen and R. Mikkelsen, "On the validity of the blade element momentum theory," in Proceedings of the European Wind Energy Conference and Exhibition, pp. 362-366, Copenhagen, 2001.

[28] G. A. M. van Kuik and L. E. M. Lignarolo, "Potential flow solutions for energy extracting actuator disc flows," Wind Energy, vol. 19, no. 8, pp. 1391-1406, 2016.

[29] R. Von Mises, Theory of Flight, McGraw-Hill, New York, NY, USA, 1945. 


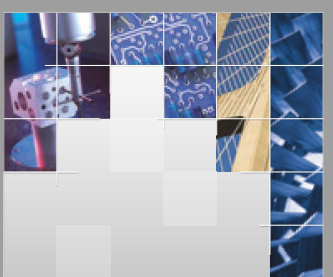

\section{Enfincering}
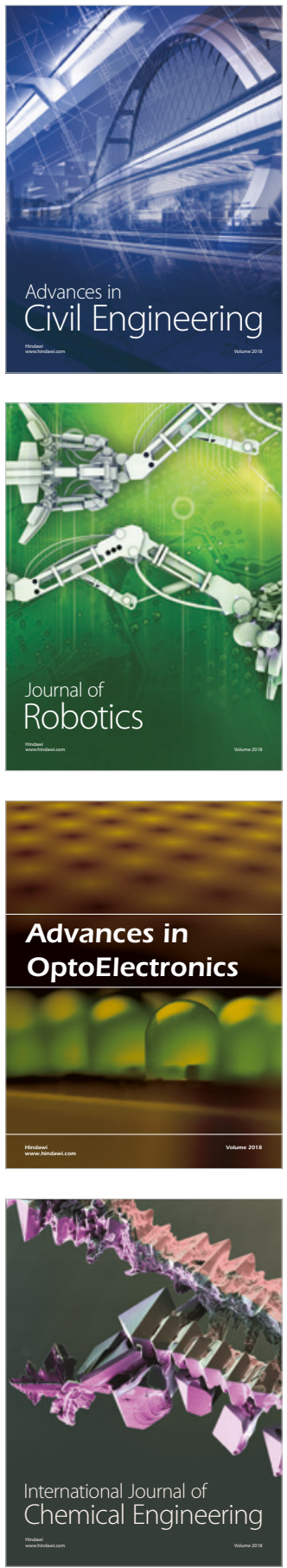

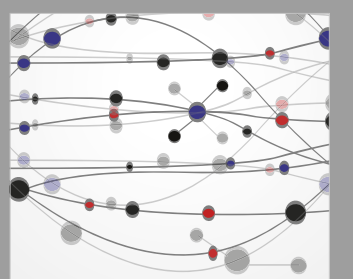

\section{Rotating \\ Machinery}

The Scientific World Journal

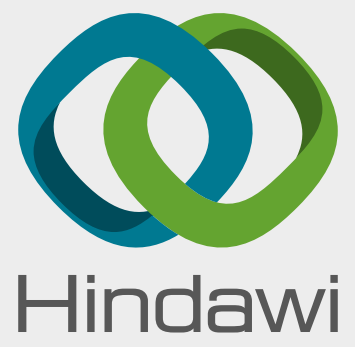

Submit your manuscripts at

www.hindawi.com
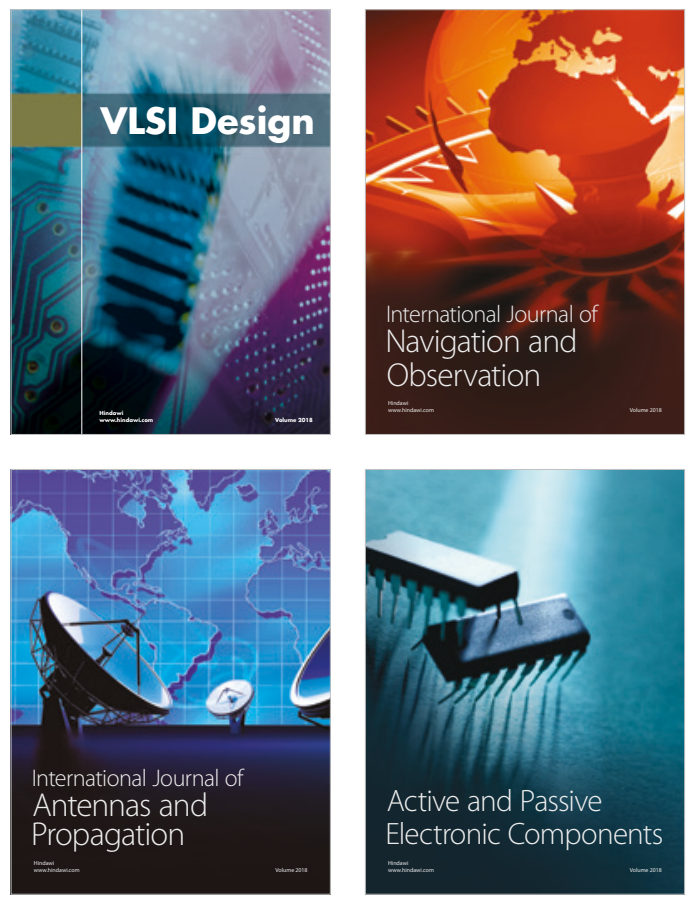
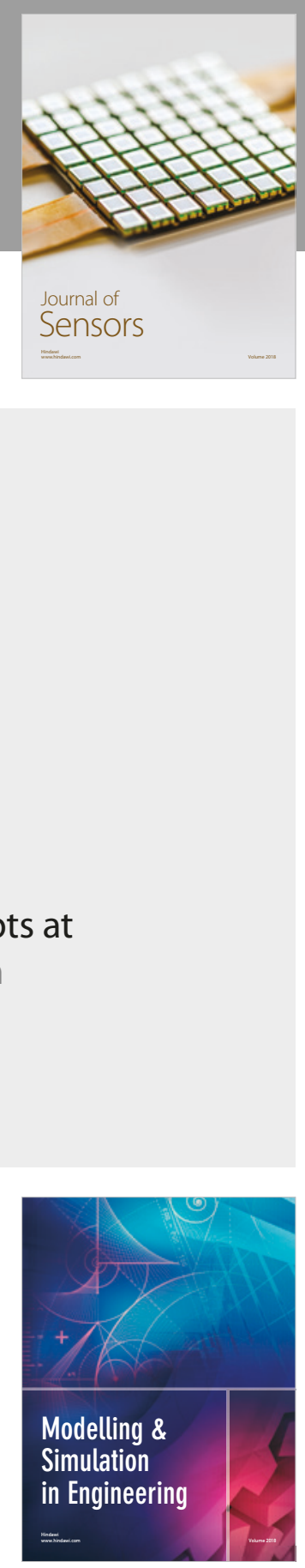

\section{Advances \\ Multimedia}
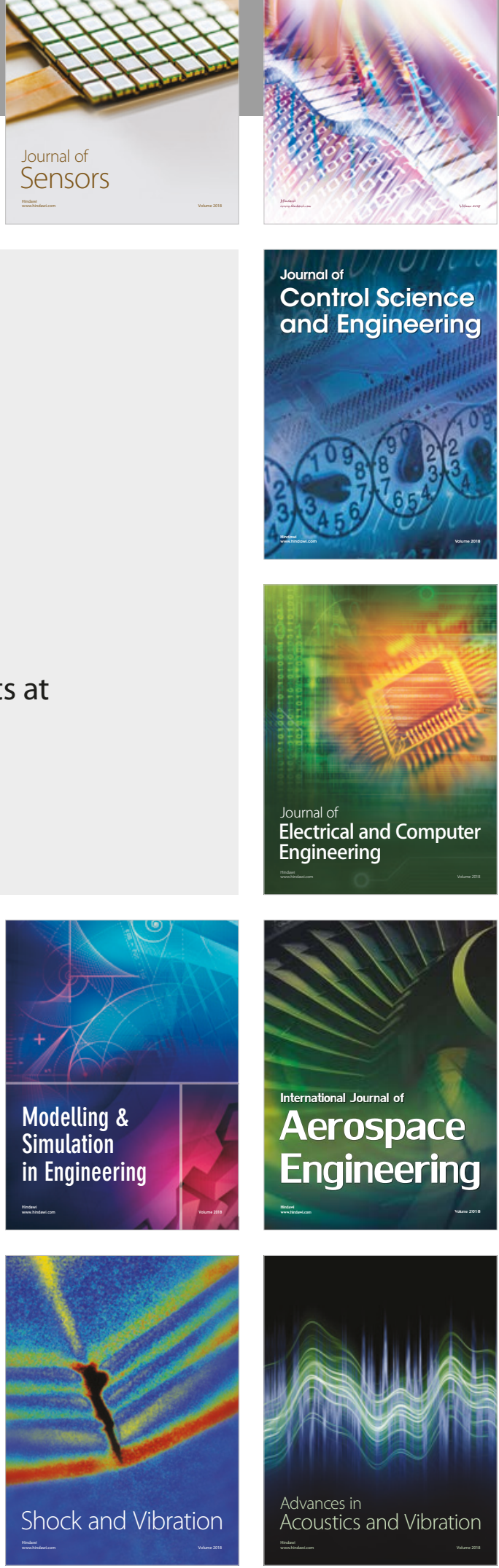